\title{
Giant Liposarcoma Neck: A Case Report
}

\author{
Dr.Dhruv Gupta ${ }^{1}$ Dr.Gurpreet Singh ${ }^{2}$ Dr.KS Mehta ${ }^{3}$ Dr.Deepika Sharma ${ }^{4}$ \\ 1. Postgraduate Student Department Of Surgery ASCOMS And Hospitals Jammu (J\&K) India \\ 2. Assistant Professor Department Of Surgery ASCOMS And Hospitals Jammu (J\&K) India. \\ 3. Professor Department Of Surgery ASCOMS And Hospitals Jammu (J\&K) India. \\ 4. Postgraduate Student Department Of Surgery ASCOMS And Hospitals Jammu (J\&K) India.
}

\begin{abstract}
Liposarcoma is a malignant mesenchymal neoplasm that arises from the adipose tissue. Liposarcomas account for 35\% to $45 \%$ of all soft tissue sarcomas. Liposarcomas are reported in the extremities and retroperitoneum [1] but the neoplasm rarely arise from head and neck region. Only about 2\% of liposarcomas present in the head and neck region and as such there is a limited reporting of these tumors in the literature. Authors are describing a rare case of "Giant Liposarcoma Neck" rare both in terms of site ie. neck and size ie. giant liposarcoma.
\end{abstract}

Keywords: Giant, Liposarcoma, Mesenchymal, Neck, Retroperitoneum .

\section{Introduction:}

Liposarcoma is a rare cancer of connective tissues that resemble fat cells under microscope. These tumors are most likely to arise from deep-seated structures mostly retroperitoneum or in some cases from lower extremities rather than from submucosal or subcutaneous fat which are rare entities. There is a male predominance and etiologic factors include Nf-1 gene, trauma and irradiation [2]. Liposarcoma tends to affect adults between the ages of 40yrs and 60yrs. The gross appearance of the tumor depends on the histologic type, degree of vascularity, presence of necrosis, and amount of mature fat and fibrous tissue. Surgery is the treatment for primary liposarcomas that have not yet spread to other organs. In most cases, a surgeon will remove the tumor, along with a wide margin of healthy tissue around the tumor [figs3] however in head and neck region compartmental resection, which is possible in the extremities is restricted by vital neurovascular structures, thus postoperative Radiotherapy is frequently undergone. Five-year survival was $100 \%$ for well-differentiated, $73 \%$ for myxoid, $42 \%$ for pleomorphic, and $0 \%$ for round cell liposarcomas [3][4].

\section{Case Presentation:}

Authors report a case of 62 yrs old male patient resident of kathua ,jammu labourer by profession who reported to our centre for management of a giant tumourous growth on right side of the neck that gradually developed over a period of 2 yrs. Patient was alright 2 yrs back when he incidentally noticed a small swelling on right side of the neck which gradually progressed in size over a period of 2 yrs to its present size . Cosmetic consideration and decreased function (ie, range of motions) of neck were the only complaints for which the patient wanted to get treated. Swelling was painless not associated with any discharge . No history of difficulty in speech, swallowing and breathing. There was also no history of diabetes mellitus ,hyper/hypotension ,hyper/hypothyroidism ,tuberculosis or similar swelling in the past .No similar complaints in any other family member in past.

On clinical examination there was a single big globular pedunculated swelling approximately $16 \mathrm{~cm}$ vertically and $28 \mathrm{~cm}$ horizontally [figs2].It extended superiorly upto the tip of mastoid bone, inferiorly upto the middle of the clavicle, medially upto middle of the anterior border of sternocleidomastoid and posteriorly upto the external occipital protuberance. It was variable in consistency with both soft and hard components within the same swelling.It was non tender,non pulsatile mobile both horizontally and vertically, margins well defined and over lying skin normal with smooth surface.Both transillumination and fluctuation test were negative No bruit was noted on auscultation and regional lymph nodes were not enlarged.Oral cavity and oropharynx were normal.

\section{Indirect Laryngoscopy:}

1.Epiglottis and Aryepiglottic fold- Normal

2.Prominent ventricular folds

3.Vocal Cords -Size,Shape,Mobility-Normal

4.Anterior Commissure-Normal 
FNAC: Smear shows moderate cellularity,oval to spindle shaped nuclei in myxomatous background. Ill defined cytoplasm.Moderate nuclear pleomorphism. Occasional bizzare nuclei with capillaries criss crossing the lesion.Mast cells are present in the myxoid stroma.Myxoid soft tissue neoplasm most likely low grade sarcoma.

CECT: Heterogenous enhancing mass lesion and irregularly enhancing wall with internal necrosis in right side of the neck superficial to sternocleidomastoid with stranding of subcutaneous fat planes.

\section{CECT NECK [figs1]}
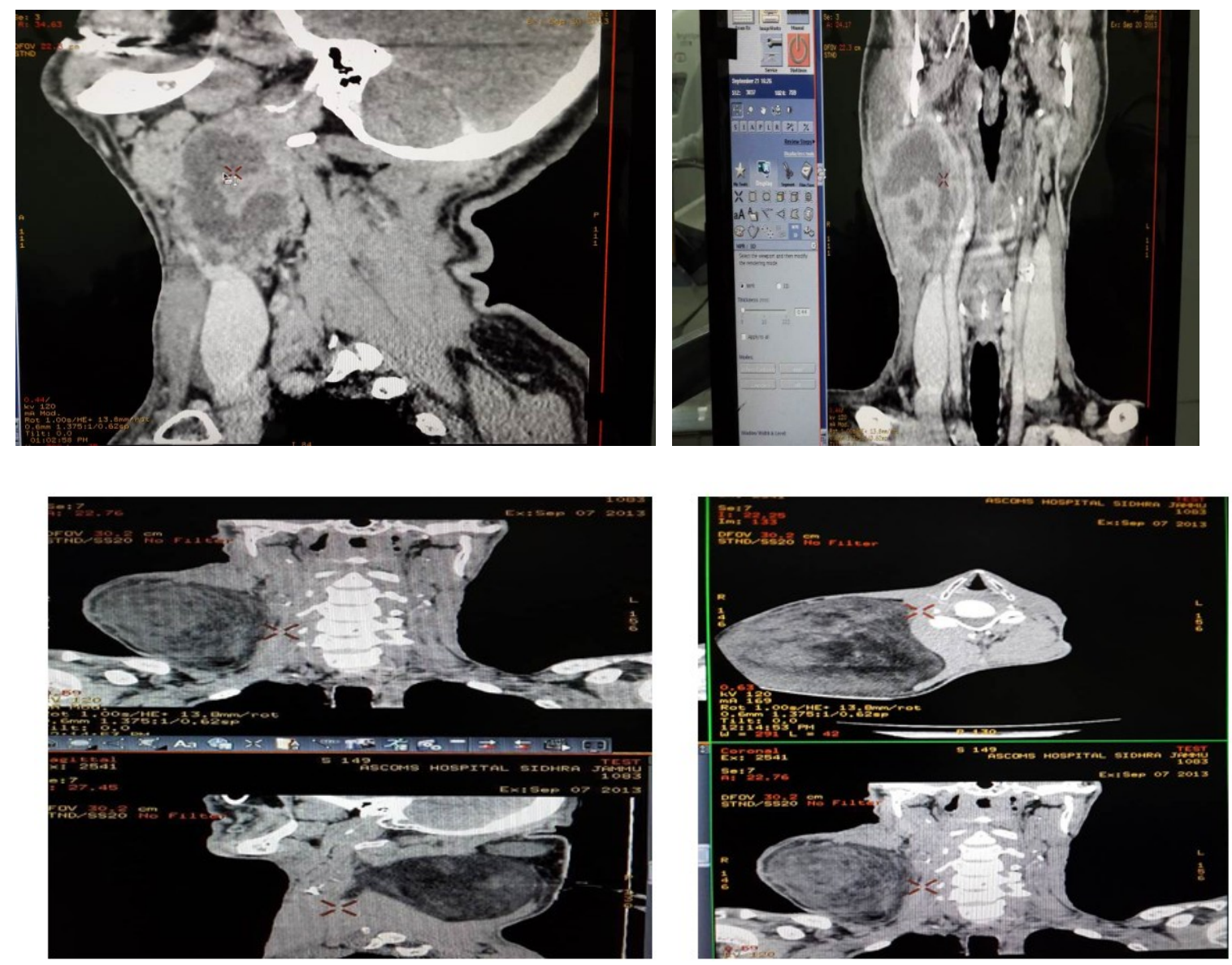

\section{Discussion:}

Liposarcoma is a malignancy of fat cells. Liposarcoma normally appears as a slowly enlarging, painless, non ulcerated submucosal mass in a middle-aged person, but some lesions grow rapidly and become ulcerated early. The development of a liposarcoma from a preexisting benign lipoma is rare. Most cases arise de novo. Liposarcomas most frequently arise from the deep-seated stroma rather than the submucosal or subcutaneous fat. The most recent World Health Organization classification [5] of soft tissue tumors recognizes 5 categories of liposarcomas: (I) well differentiated, which includes the adipocytic, sclerosing, and inflammatory subtypes; (II) dedifferentiated; (III) myxoid; (IV) round cell; and (V) pleomorphic .A spindle-cell variant of welldifferentiated liposarcoma is also described. The advent of cytogenetic and molecular investigations has contributed to better categorization of this subset of mesenchymal neoplasms. Not only have they provided new insights into the biology of these tumors, but they have also validated the current classification schemes based on conventional morphologic observations. In rare circumstances, lesions can have a combination of morphologic types; these are classified as combined or mixed-type liposarcomas. The anatomical distribution of liposarcoma appears to be partly related to the histologic type. Well-differentiated liposarcoma tends to occur in deep soft tissues of both the limbs and the retroperitoneum. Myxoid and/or round-cell liposarcomas and pleomorphic liposarcomas have a striking predilection for the limbs, and dedifferentiated liposarcoma occurs predominantly in the retroperitoneum. Liposarcoma subtype arising from the subcutaneous tissue of the skin of the neck appears to be exceedingly rare. Fusion proteins created by chromosomal abnormalities are key components of mesenchymal cancer development. An abnormality of band 12q13 has been associated with the development of liposarcomas. The most common chromosomal translocation is the FUS-CHOP fusion gene, 
which encodes a transcription factor necessary for adipocyte differentiation. Myxoid liposarcoma is also notable for metastasizing to unusual sites, including the serosal surfaces of the pleura, mediastinum, pericardium, and diaphragm,and to extrapulmonary soft-tissue sites, including the peritoneum, chest wall, and breast. Lymph node metastases are extremely rare. The rationale for wide surgical excision [figs3] is the prevention of recurrence and dedifferentiation. Wide and deep surgical excision, along with local radiation and/or chemotherapy, may be necessary for high-grade lesions[6][7]. Factors that predict a better prognosis for liposarcomas are a well differentiated and myxoid histology, a low percentage of the round-cell component, no spontaneous necrosis, a low number of mitoses, a size of less than $5 \mathrm{~cm}$ and age less than 45 years. Although grossly these tumors appear to be encapsulated, they extend by infiltration; the likelihood of nearby satellite nodules necessitates wide excision Given the favorable outcome with wide surgical excision alone, regardless of the histologic type of the tumor[8], some authors believe that adjuvant radiation therapy is unjustified.

\section{PREOPERATIVE IMAGES [figs 2]}
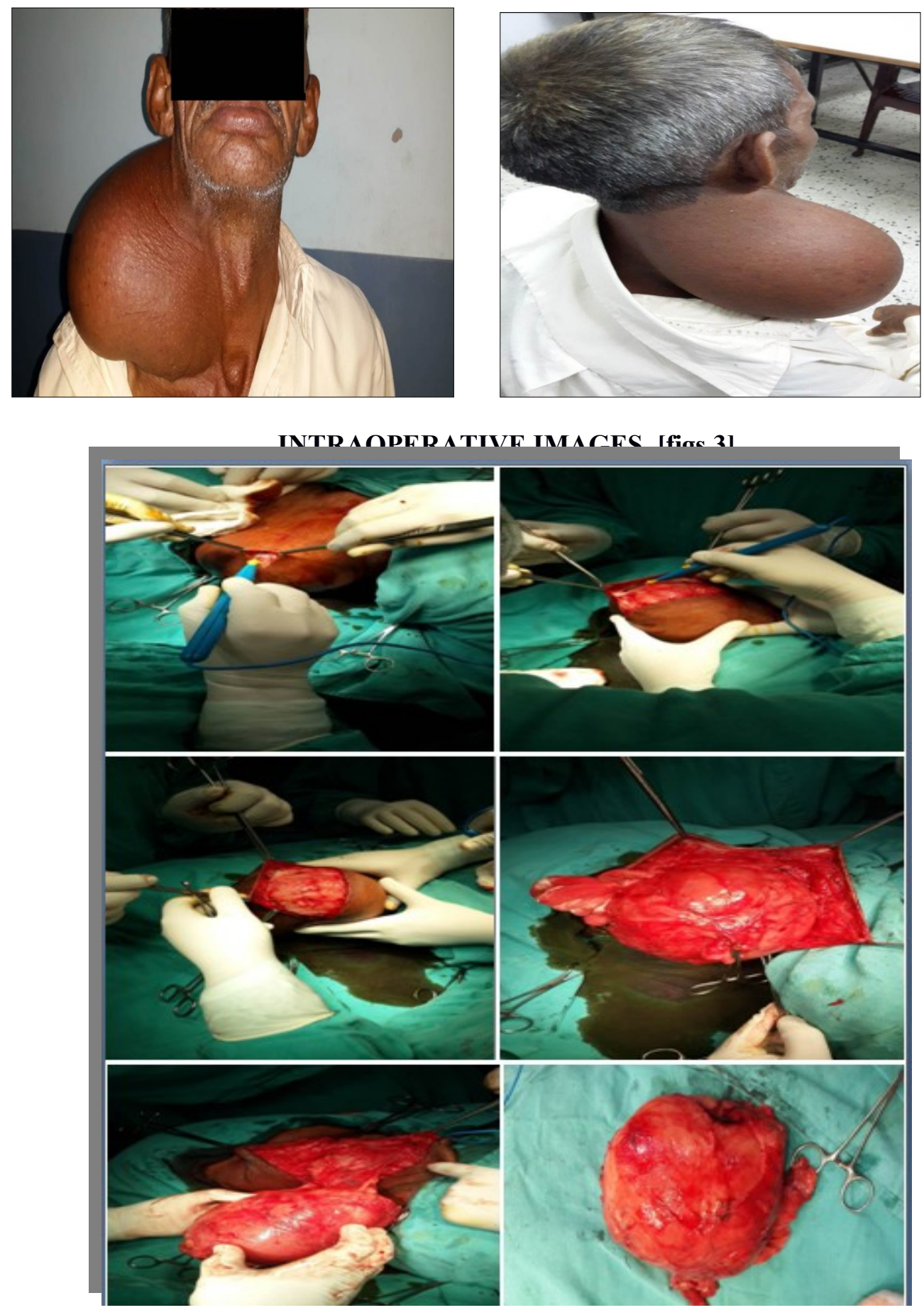

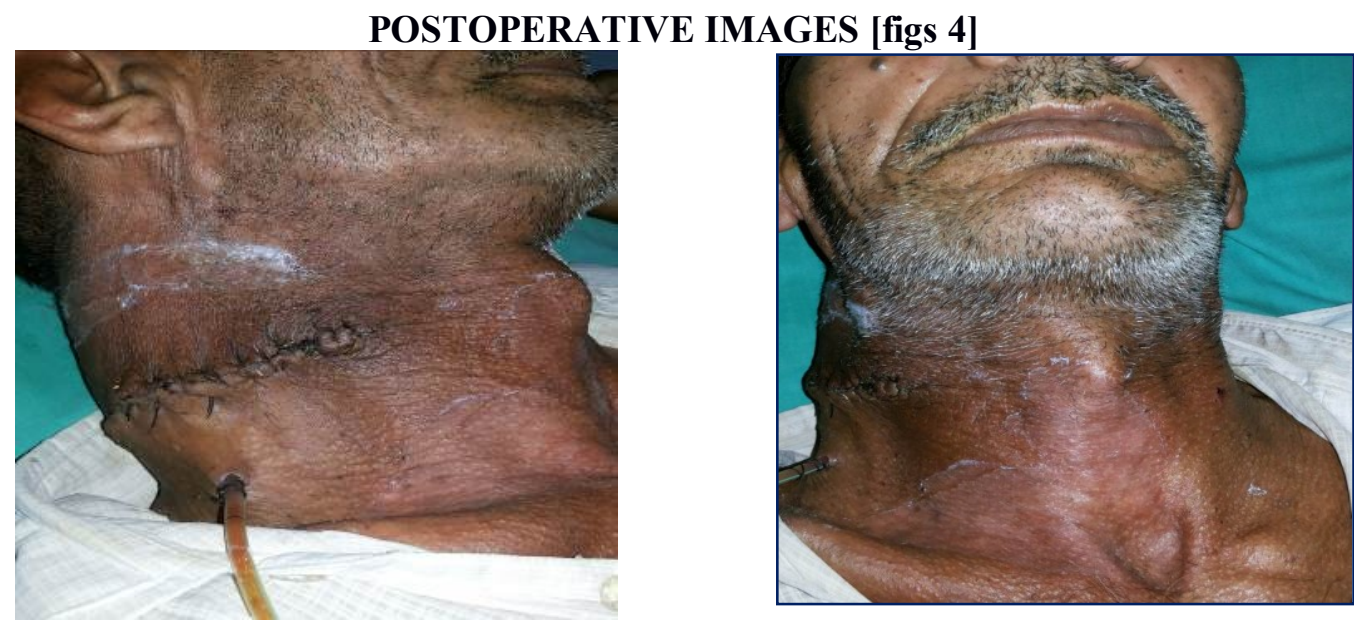

HISTOLOGY [fig5]

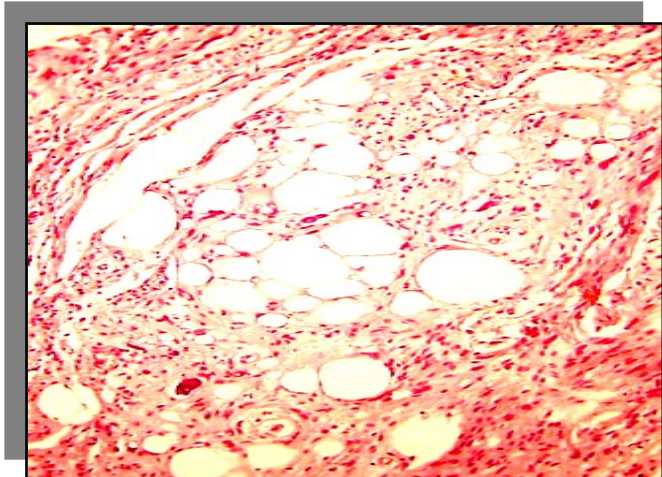

\section{Conclusion:}

Giant Liposarcoma neck is a rare mesenchymal malignant tumour. Clinically and radiologically it may be confused with other benign or malignant tumours. Histopathological examination is important not only for accurate diagnosis of this but also for appropriate treatment. Although surgical resection is the mainstay of curative treatment, patients may benefit from multimodality treatment with adjuvant radiotherapy.

\section{Acknowledgments: \\ Dr Deepika Sharma \\ PG Surgery ASCOMS \\ All financial support for the study was done by \\ Dr Dhruv Gupta and Dr Deepika Sharma}

\section{Ethical Approval Statement:}

Written informed consent was obtained from the patient for publication of this case report and accompanying images. A copy of the written consent is available for review by the Editor-in-Chief of this journal on request.

\section{References:}

[1]. Peterson JJ, Kransdorf MJ, Bancroft LW, O’Connor MI. Malignant fatty tumors: classification, clinical course, imaging appearance and treatment. Skeletal Radiology 2003; 32: pp 493-503.

[2]. Enzinger FM and Weiss SW eds. General considerations In Soft Tissue Tumors, Third Edition. St. Louis, 1995, Mosby, pp 1 -16.

[3]. Robinson E, Neugut AI, Wylie P. Clinical aspects of postirradiation sarcomas. J Natl Cancer Inst 1988; 80: 233.

[4]. Laskin WB, Silverman TA,. Postradiation soft tissue sarcomas: an analysis of 53 cases. Cancer 1988; 62:2330

[5]. Christopher DM, Unni KK, Mertens F. WHO classification of tumors. Pathology and genetics: tumors of soft tissue and bone. Lyon, France, 2002, IARC Press, pp 35-46.

[6]. Zagars GK, Goswitz MS, Pollack A. Liposarcoma: outcome and prognostic factors following conservation surgery and radiation therapy. Int J Radiat Oncol Biol Phys 1996; 36:311-319

[7]. Spiro IJ, Gebhardt MC, Jennings C et al. Prognostic factors for local control of sarcomas of the soft tissues managed by radiation and surgery. Seminars in Oncology 1997; 24(5): pp 540-546.

[8]. McCulloch TM, Makielski KH, McNutt MA: Head and neck liposarcoma. A histopathologic reevaluation of reported cases. Arch Otolaryngol Head Neck Surg 118:1045-1049, 1992. 